\title{
Radium-223 dichloride: a new paradigm in the treatment of prostate cancer
}

\author{
Urbano Anido Herranz ${ }^{1}$, Ovidio Fernández Calvo ${ }^{2}$, Francisco Javier Afonso Afonso ${ }^{3}$, \\ Sofía Rodríguez Martínez de Llano ${ }^{4}$, Martín Lázaro Quintela ${ }^{5}$, Luis León Mateos ${ }^{1}$, \\ Sergio Vázquez Estévez ${ }^{6}$ and Luis Miguel Antón Aparicio ${ }^{7}$
}

${ }^{1}$ Medical Oncology Department, Complexo Hospitalario Universitario de Santiago de Compostela, Santiago de Compostela 15706, Spain

${ }^{2}$ Medical Oncology Department, Complexo Hospitalario Universitario de Ourense, Ourense, Spain

${ }^{3}$ Medical Oncology Department, Hospital Arquitecto Marcide de Ferrol, Ferrol, Spain

${ }^{4}$ Nuclear Medicine Department, Centro Oncológico de Galicia, A Coruña, Spain

${ }^{5}$ Medical Oncology Department, Complexo Hospitalario Universitario de Vigo, Vigo, Spain

${ }^{6}$ Medical Oncology, Hospital Universitario Lucus Augusti Lugo, Lugo, Spain

${ }^{7}$ Medical Oncology Department, Complexo Hospitalario Universitario A Coruña, A Coruña, Spain

\begin{abstract}
Radionuclides have been widely used for cancer treatment. Recently, new research about radium-223 dichloride has been conducted in prostate cancer, which reveals that it is the first radiopharmaceutical to demonstrate an improvement in overall survival and time to first symptomatic skeletal event in patients with castration resistant prostate cancer with symptomatic bone metastases. This fact has created a new paradigm in the treatment of prostate cancer landscape, where only chemotherapy and hormone therapy had a role, while $\beta$-emitters had been confined exclusively to the role of pain relief with no impact on survival. The aim of this review is to outline current treatment approaches for advanced prostate cancer with a focus on the role of radium-223 dichloride, reviewing patients' profile that make them suitable to therapy and chances for further studies.
\end{abstract}

Keywords:

alpha-emitters; bone metastases; prostate cancer; radiopharmaceuticals; radium-223 
The skeleton is the most common organ to be affected by metastatic cancer, and prostate tumors have a special propensity to spread to the bone [1]. Most patients developing castration-resistant prostate cancer (CRPC) present radiographic evidences of bone metastases and related severe pain, neurological symptoms, pathological bone fractures, hypercalcemia, spinal cord compression or pancytopenia [2]. These symptoms induce a considerable impact on quality of life due to suffering, reduced functional capacity and increased non-self-sufficiency [3,4]. Skeletal metastases from prostate cancer are predominantly bone forming or osteoblastic lesions, although osteolytic lesions have also been reported [3]. Prostate cancer cells stimulate osteoblastic proliferation to produce specific growth factors for osteoblasts, which results in increased bone matrix deposition in the bone and tumor microenvironment [3]. Current treatments for bone metastases in CRPC patients include systemic chemotherapy, hormonal therapies, radium-223 dichloride (Ra-223) and other bone-targeted therapies. Others, including externalbeam radiotherapy and analgesics, have a role in pain relief.

Unlike chemotherapy and hormonal treatments, the current bone-targeted therapies (e.g., bisphosphonates and denosumab) are mainly limited to delay the onset of skeletalrelated events (SRE) [5-7], without any improvement in survival. Similarly, externalbeam radiotherapy is an effective and costefficient treatment for metastatic bone pain, able to induce a quick symptomatic relief in 50-80\% of patients, but without any evidence of increasing overall survival (OS) [8].

The idea of using systemic radionuclide therapy targeting bone metastases results in an effective strategy able to deliver high doses of radiation to metastatic bone lesions, limiting the toxicity on healthy tissues [9-11]. During the last years, bone-targeting radiopharmaceuticals were represented by radioactive isotopes emitting b-particles, and lately by conversion electron emitters [12,13]. At present, only three compounds, ${ }^{89} \mathrm{Sr}$ (approved in Europe for prostate cancer), ${ }^{153} \mathrm{Sm}$-lexidronam (153Sm- EDTMP; approved in Europe for osteoblastic metastases) and 186Re-etidronate (186Re- HEDP; approved in some European countries) have been available for pain relief secondary to skeletal metastases [14,15]. Unfortunately, the exposure of surrounding tissues to $\beta$ and $\gamma$-emissions is associated with toxicities due to the relatively long range of the radiation. On the other hand, preliminary studies reported that radionuclides emitting $\alpha$ particles could represent a new approach in this setting [14]. $\alpha$-Emitters are characterized by shorter range $(<0.1 \mathrm{~mm})$ and higher energy radiation than $\beta$-particles and $\gamma$-rays, according to low linear energy transfer (LET) radiation. In addition, tissue penetration with $\alpha$-emitters accounts for a diameter of $2-10$ cells, minimizing the damage to healthy hematopoietic tissue. Besides, due to the high LET radiation, $\alpha$ particles are evaluated as more lethal, inducing nonrepairable double-strand DNA breaks in adjacent tumor cells [16].

Radium is a calcium mimetic bone seeker $\alpha$-particle emitter able to focus on areas of increased bone turnover [14]. Presently, two forms of radium are available for clinical practice, Ra-223 and Ra-224. While Ra-224 has been used to treat only ankylosing spondylitis since 1999 [17], Ra-223 has been the first $\alpha$-emitter radiopharmaceutical available for bone metastases treatment secondary to CRPC [18]. In addition, Ra-223 has been demonstrated to increase OS in a Phase III study involving CRPC patients with bone metastases, thus indicating that Ra-223 should not only be evaluated as a simple bone-seeking radiopharmaceutical, but also as a systemic strategy for these patients [19].

The aim of this review article is to outline the clinical development of Ra-223, analyzing the rationale and evaluating the main toxicities associated with the therapy. In addition, we will discuss the appropriate patient profile that makes a patient suitable for treatment with Ra-223 in the context of the new therapeutic landscape. 


\section{Radium-223}

\section{Biophysical \& preclinical studies}

Biophysical studies indicated that Ra-223 is a bone-seeking $\alpha$-emitter agent that, due to the similar structure of calcium, is able to localize in the high calcium turnover, resorption, and formation areas such as bone metastasis [20]. These studies revealed that Ra-223 generates highly localized radiation in bone metastases of non-repairable double-stranded DNA breaks with minimal effects on normal tissues $[19,21,22]$.

Preliminary in vitro studies performed in cervical carcinoma cell line nhIK 3025 showed that, after the exposure to different doses of Ra-223, a radiation dose of 0.35 Gy induced a permanent stop in the G2 Phase of the cell cycle [20].

Subsequently, extensive preclinical studies conducted in animal models confirmed that Ra-223 acts as calcium mimetic naturally binding to the areas of bone formation [17,21,23-25].

In particular, studies conducted on mice revealed stability and affinity for the $\alpha$-emitter with osseous tissue, with the possibility of delivering the therapeutic radiation to bone metastases with acceptable bone marrow toxicity [25]. Following dosimetric evaluations indicated lower bone marrow toxicity for Ra-223 versus mice treated with $89 \mathrm{Sr}$, due to the lower uptake of Ra-223 in bone marrow (1\%) after 3 days from the administration [25].

Further studies conducted in a human breast cancer model (MT1) and animal models with bone cancer confirmed that, when Ra-223 is administered intravenously, it accumulates in the whole bone tissue, with a more intense concentration in high turnover areas as trabecular bone, fractures or metastases [21,23].

\section{Ra-223 \& radioprotection}

Ra-223 has a half-life of 11.4 days, in between $\beta$-emitter treatments $\left({ }^{89} \mathrm{Sr}: 50.5\right.$ days, ${ }^{153} \mathrm{Sm}$-EDTMP: 1.9 days) [15], what is an advantage for its preparation, transportation and administration, being also short enough to avoid the generation of longlived radioactive waste in the hospital [20]. Most of the emitted energy is comprised of $\alpha(95.3 \%), \beta(3.6 \%)$ and $\gamma(1.1 \%)$ particles or x-rays [20]. The short penetration of the $\alpha$ particles allows the use of standard radiation protection measures during shipping and administration; minimal radiation shielding is required.

Alpha particles induce non-repairable double-stranded DNA breaks in adjacent tumor cells [16]. These particles are classified as high LET radiation with a low range of action $(<100 \mathrm{~m})$, minimizing damage to healthy tissue surrounding cancer cells. In contrast, $\beta$ particles from therapeutic agents used for the palliation of pain $\left({ }^{89} \mathrm{Sr}\right.$ and ${ }^{153} \mathrm{Sm}$-EDTMP) produce low-LET radiation with a greater range of action and therefore myelosuppression [25]. Alpha particle emitters locate cellular destruction with high-dose radiation in a smaller area with minimal bone-marrow toxicity.

The administered activity is absorbed by the bone in the initial hours after the intravenous administration (TABLE 1). The major route of elimination is intestinal [24], within the first week of treatment and to a lesser extent through the urine, contrary to other radionuclide therapies [21]. This seems to explain its main non-hematologic side effects, mainly digestive that, although manageable, limits its use in patients with fecal incontinence. The most frequently observed adverse reactions $(\geq 10 \%)$ in patients receiving Ra-223 were diarrhea, nausea, vomiting and thrombocytopenia [26]. 
Table 1. Calculated absorbed radiation dose to organs radium-223.

\begin{tabular}{lllll}
\hline $\begin{array}{l}\text { Target organ } \\
(\mathrm{Gy} / \mathrm{MBq})\end{array}$ & $\begin{array}{c}\alpha \text { emission } \\
(\mathrm{Gy} / \mathrm{MBq})\end{array}$ & $\begin{array}{c}\beta \text { emission } \\
(\mathrm{Gy} / \mathrm{MBq})\end{array}$ & $\begin{array}{l}\gamma \text { emission } \\
(\mathrm{Gy} / \mathrm{MBq})\end{array}$ & $\begin{array}{l}\text { Total dose } \\
(\mathrm{Gy} / \mathrm{MBq})\end{array}$ \\
\hline & & & & \\
Lower large intestine wall & 0 & 0.04560 & 0.00085 & 0.04645 \\
Small intestine wall & 0.00319 & 0.00360 & 0.00047 & 0.00726 \\
Stomach wall & 0 & 0.00002 & 0.00012 & 0.00014 \\
Upper large intestine wall & 0 & 0.03150 & 0.00082 & 0.03232 \\
Kidneys & 0.00299 & 0.00011 & 0.00011 & 0.00320 \\
Liver & 0.00279 & 0.00010 & 0.00008 & 0.00298 \\
Red marrow & 0.13200 & 0.00642 & 0.00020 & 0.13879 \\
Osteogenic cells & 1.14000 & 0.01490 & 0.00030 & 1.15206 \\
Urinary bladder wall & 0.00371 & 0.00016 & 0.00016 & 0.00403 \\
Whole body & 0.02220 & 0.00081 & 0.00012 & 0.02311 \\
& & & & \\
\hline
\end{tabular}

Calculations of absorbed doses were performed using OLINDA/EXM (Organ Level INternal Dose Assessment/EXponentialModeling), a software based on the Medical Internal Radiation Dose algorithm, which is widely used for established beta- and gamma-emitting radionuclides.

Gy: Grays; MBq: Megabecquerel.

Regarding administration, it is performed by slow intravenous injection, and only by persons authorized to handle radiopharmaceuticals in designated clinical settings and after evaluation of the patient by a qualified physician. The volume to be administered should be calculated using the patient's body weight $(\mathrm{kg})$, dosage level $(50 \mathrm{kBq} / \mathrm{kg}$ body weight), radioactivity concentration of the product (1000 $\mathrm{kBq} / \mathrm{ml}$ ) at reference date (the reference date is stated on the vial and lead pot label) and decay correction (DK) factor to correct for physical decay of Ra-223 (a table of DK factors is provided with each vial as part of the booklet). The amount of radioactivity in the dispensed volume shall be confirmed by measurement in a properly calibrated activimeter. The total volume to be administered to a patient is calculated as follows: Volume to be administered $(\mathrm{ml})=$ Body weight $(\mathrm{kg}) \times$ activity $(50 \mathrm{kBq} / \mathrm{kg}$ body weight)/DK factor $\times 1000 \mathrm{kBq} / \mathrm{ml}$. No dose adjustment is considered necessary in patients with renal or hepatic impairment.

Special warnings and precautions should be considered in patients with bone-marrow suppression. Therefore, hematological evaluation must be performed at baseline and prior to every dose of Ra-223. Before the first administration, the absolute neutrophil count should be $\geq 1.5 \times 109 / 1$, the platelet count $\geq$ $100 \times 109 / 1$ and hemoglobin $\geq 10.0 \mathrm{~g} / \mathrm{dl}$. Before subsequent administrations, the absolute neutrophil count should be $\geq 1.0 \times 109 / 1$ and the platelet count $\geq 50 \times 109 / 1$.

Spinal cord compression and bone fractures should be treated as clinically indicated before starting treatment with Ra-223. In patients treated with bisphosphonates and Ra-223, an increased risk for developing osteonecrosis of the jaw cannot be excluded. In the Phase III study, cases of osteonecrosis of the jaw were reported in $0.67 \%$ patients (4/600) in the Ra-223 arm compared to $0.33 \%$ patients $(1 / 301)$ in the placebo arm. However, all patients with osteonecrosis of the jaw were also exposed to prior or concomitant bisphosphonates (e.g., zoledronic acid) and prior chemotherapy (e.g., docetaxel) [27]. No cases of Ra-223-induced cancer have been reported in clinical trials in follow-up of up to 3 years.

The external radiation exposure associated with handling of patient doses is considerably lower in comparison to other radiopharmaceuticals for therapeutic purposes as the administered radioactivity will usually be below $8 \mathrm{MBq}$. Ra-223 contamination is highly unlikely [23]. This justifies the absence of patients' contact restrictions with others after the treatment is administered. 


\section{Review of Phase I-III clinical trials}

\section{Phase I}

Based on the promising preclinical results, a Phase I dose escalating and safety study of Ra-223 was conducted in 25 subjects (15 metastatic CRPC and 10 metastatic breast cancers). The primary objective was to estimate the radiation dosimetry of a single dose of Ra-223. Patients were injected with 46-250 $\mathrm{kBq} / \mathrm{kg}$ intravenously and were followed for 8 weeks for adverse events (AEs) monitoring [28]. Some reversible myelosuppression occurred, with nadirs 2-4 weeks after injection and complete recovery during the follow-up period. The incidence and severity of all toxicities were directly related to the administered dose. The most common adverse reactions were diarrhea, bone pain (flare reaction included), asthenia, nausea and vomiting. Potentially serious side effects included diarrhea (one patient) and leucopenia (two patients). There were no hematological dose-limiting toxicities up to the maximum planned dose.

The feasibility of repeating Ra-223 injections of two fixed dosage levels administered according to the schedule of 3- or 6-week intervals was evaluated in a Phase Ib study [29]. Six patients were recruited and received a total dose of $250 \mathrm{kBq} / \mathrm{kg}$, two of them received injections of $125 \mathrm{kBq} / \mathrm{kg}$ at 6 -week intervals, while four patients received $50 \mathrm{kBq} / \mathrm{kg}$ given five-times at 3-week intervals.

Twenty out of the 31 patients included in the Phase I studies were still alive 1 year after the first injection of Ra- 223. No hematological, renal, gastrointestinal or hepatic late toxicities were reported. The consistent reduction in bone alkaline phosphatase (BAP) levels suggest that the most strongly targeted areas by Ra-223 would be the regions with an elevated bone metabolism, as is often seen in the zones of developing skeletal metastases [29]. The decrease of the serum BAP, as surrogate marker, and the relief of secondary pain in bone metastases encouraged to perform the following Phase II studies.

\section{Phase II}

The placebo-controlled Phase II study BC1-02 was conducted in patients with symptomatic CRPC [30]. Patients with bone pain needing external beam radiotherapy were assigned to four intravenous injections of Ra-223 (50 kBq/kg) or placebo, every 4 weeks for 12 weeks. The primary endpoints were the mean change in BAP from baseline to 4 weeks after the last injection and time to occurrence of SREs. Secondary endpoints included evaluation of safety, serum markers of bone turnover, prostate specific antigen (PSA) and OS. No differences in toxicity, except constipation, were reported, and hematological toxic effects did not differ significantly between the two groups, with no patients discontinuing Ra-223 because of treatment toxicity. Similar to Phase I trials, the results reported a decrease in PSA of over 50\% from baseline in patients treated with Ra-223. Median time to PSA progression was 26 weeks versus 8 weeks for Ra-223 versus placebo, and median time to first SRE was 14 weeks in the Ra-223 arm versus 11 weeks in the placebo arm ( $\mathrm{p}=0.257)$, with a hazard ratio $(\mathrm{HR})$ of $1.75(95 \% \mathrm{CI}: 0.96-3.19 ; \mathrm{p}=0.065)$ favoring Ra-223 when adjusted for baseline covariates. A significant OS benefit was reported, although, bias may exist with regard to these data due to the relatively small number of included patients (64 in total) and the concomitant treatment with steroids or antiandrogens in these patients [30].

The BC1-03 study was a double-blinded, dose-ranging, randomized study comparing the palliative effects of four different single dose levels of Ra-223 $(5,25,50$ or $100 \mathrm{kBq} / \mathrm{kg})$ in progressive metastatic CRPC patients with bone metastases and pain [18]. The primary endpoint was the change in 'pain index' (derived from a combination of the visual analog scale and analgesic consumption categorized according to the WHO analgesic use score at weeks 2, 4, 8, 12 and 16). Secondary endpoints included change from baseline in the brief pain inventory severity index and functional index, OS, duration of pain relief, relative change in bone BAP and PSA and assessment of AEs. One hundred patients were randomized. A statistically significant dose response, for pain relief and stable analgesic use, occurred at week 2 ( $\mathrm{p}=$ 0.035 ). At week 8 , there were $40,63,56$ and $71 \%$ patients with pain relief in the $5,25,50$ and $100 \mathrm{kBq} / \mathrm{kg}$ 
groups, respectively [18]. BAP decrease over 50\% from baseline occurred in all groups, although it was only statistically significant in the $100 \mathrm{kBq} / \mathrm{kg}$ dose group at weeks 4 and 8 . A well-tolerated safety profile was observed. Remarkably, there may have been a problem with dropout bias, since 17 patients dropped out by week 8 , and there was an important difference in dropouts in the low versus high-dose arms (eleven vs six patients).

The BC1-04 was a multicenter double-blinded, randomized, dose-finding study in patients with CRPC and bone metastases. Three dose levels of Ra-223 (25, 50 and $80 \mathrm{kBq} / \mathrm{kg})$ were compared, every 6 weeks for 12 weeks (three total doses). The primary end point was PSA response ( $\geq 50 \%$ decline from baseline); bone markers, SREs, AEs, and survival were secondary endpoints [31]. A total of 121 patients were randomized. Primary endpoint was achieved, showing a dose-dependent effect for the three dose levels 25,50 and $80 \mathrm{kBq} / \mathrm{kg}$, respectively. Again, the good safety profile of Ra-223 was confirmed. The most frequent AEs were nausea, vomiting and diarrhea and two cases of grade 3-4 thrombocytopenia were observed [31]. Normalization of BAP was associated with a significantly better survival, compared with those patients who did not achieve BAP normalization. Observed data from BC1-04 study supported the optimal therapeutic dose level of $50 \mathrm{kBq} / \mathrm{kg}$ for a subsequent Phase III trial.

\section{Phase III}

The ALSYMPCA trial was an international, randomized, double- blinded, placebo-controlled Phase III study conducted in men with symptomatic metastatic CRPC [19,32,33]. Inclusion criteria included symptomatic CRPC, two or more bone metastases, no known visceral metastases and either docetaxelpretreated patients or those that were unfit for docetaxel. Patients were randomized 2:1 to receive Ra-223 injections at $50 \mathrm{kBq} / \mathrm{kg}$ plus best standard of care versus placebo plus best standard of care. 'Best standard of care' included secondary hormonal therapies or external-beam radiotherapy, but not cytotoxic chemotherapy or radioisotopes. Subjects were stratified according to total BAP, bisphosphonate use and prior docetaxel treatment. Primary endpoint was OS, and secondary endpoints were time to first SRE, time to total BAP progression, total BAP response, total BAP normalization, time to PSA progression, safety and quality of life. The trial enrolled 922 patients (Ra-223, $n=614$; placebo, $n$ = 307). Patients enrolled in the study received up to six intravenous injections of Ra-223 or placebo, given at 4-week intervals $[19,32,33]$.

The trial was stopped based on the recommendation of an independent data monitoring committee, due to early evidence of benefit in terms of OS (with crossed predetermined boundary). Ra-223 significantly improved OS in patients with CRPC and bone metastases compared with placebo ( $p<0.001$; $\mathrm{HR}=0.70$; 95\%; CI: 0.58-0.83; median OS 14.9 months versus 11.3 months, respectively). A beneficial effect was also shown in all subgroups except for patients with Eastern Cooperative Oncology Group score (ECOG)-PS 2 or superior, which did not reach statistical significance. Time to first SRE was significantly prolonged (median 15.6 months versus 9.8 months, respectively; HR $=0.66$; $95 \%$ CI: $0.52-$ $0.83 ; \mathrm{p}<0.001)[17,19]$. In addition, there was a meaningful improvement in the quality of life, evaluated with functional assessment scale - prostate subscale scores; mainly for pain, physical, social/familial, emotional and functional well being subscales [19,33].

No differences in severe side effects (grade 3-4) were observed between Ra-223 and placebo groups. Anemia incidence was identical in both arms (13\%), as well as for neutropenia (3 vs 1\%), diarrhea and vomiting ( $2 \%$ in both arms). Likewise, toxicity of any grade (grade 1-4), showed only small differences in incidence of diarrhea (25\% of patients with Ra-223 vs $15 \%$ in patients with placebo) and thrombocytopenia (12 vs 6\%, respectively) $[19,33]$.

This study not only achieved an improvement in OS, but also in all secondary endpoints including time to first SRE. As of note, the Ra-223 arm was compared to standard treatment at physician's choice and, therefore, these interesting results may be applicable to routine clinical practice. This is particularly interesting, since other palliative therapies, such as radiotherapy, were considered as a prohibited concomitant medication in the Phase III abiraterone trial. Nonetheless, there is an important limiting 
factor in the ALSYMPCA trial: visceral metastases were an exclusion criterion, and this circumstance may appear in up to $25 \%$ of patients with CRPC $[19,34]$.

The safety results within approximately 1.5 years after treatment concluded that the incidence of myelosuppression among Ra-223 patients remained low and that there were no additional safety issues of acute myelogenous leukemia, myelodysplastic syndrome, aplastic anemia or primary bone cancer. Thus, findings from this long-term follow-up data support further evaluation of combining Ra-223 with other agents for the treatment of patients with CRPC and symptomatic bone metastases [27].

\section{Alpha emitter for CRPC patients: new opportunities}

Since Ra-223 has a mechanism of action completely different from traditional systemic therapies already available in CRPC treatment, such as chemotherapy (docetaxel and cabazitaxel) and hormone therapy (abiraterone and enzalutamide), its introduction in clinical practice could be considered a new therapeutic paradigm [31]. Therefore, a number of new issues are emerging. First, how can those patients be identified who should be treated with Ra-223 and, subsequently, what are the best tools for a correct follow-up of treated patients? And finally, what opportunities are open for future studies, such as combination with other drugs in the next future.

Once the main preclinical and clinical data are reviewed, it can be concluded that main strengths of Ra-223 include:

- It has a new and challenging mode of action and is clearly a major advance over first-generation radiopharmaceuticals.

- It is well tolerated, with easy-to-handle side effects, and with no renal or liver toxicity.

- There is a wide population that could benefit from Ra-223, since most of the patients in routine clinical practice fulfill the ALSIMPCA eligibility criteria.

- It improves survival as well as quality of life, pain control and other secondary endpoints.

- The medication dosage is comfortable; administration is simple, not time consuming and suitable for most patients. .

- Results can be generalized since 'standard of care' is a very wide option, including also radiotherapy.

With regard to weaknesses, it could be pointed that the likely ordering of treatment lines in this setting of patients is still unclear. Patients who had progressed after docetaxel treatment were eligible in the ALSYMPCA trial, and even this subgroup benefited from Ra-223. There are several potential treatment choices for these patients before switching to Ra-223. If the 'switch' is made while there are still alternative options, it brings up the question of whether chemotherapy is a comparator to Ra-223 in some situations.

\section{Patient profile}

With the purpose of describing the appropriate patient profile for Ra-223, a comparison of the baseline characteristics included in the Phase III studies of cabazitaxel, abiraterone and enzalutamide showing survival benefit in metastatic CPRC patients that had progressed after docetaxel treatment has been performed (TABLE 2) [33-36]. These results are only descriptive without any comparative aim, since the role of each only can be defined in terms of current clinical data and practice. These data are analyzed taking into consideration the efficacy reported in the subgroup analysis of every single study, keeping in mind that all these analyses were not powered to draft any conclusion. 
Table 2. Baseline characteristics of patients included in the Phase III trials with radium-223, cabazitaxel, abiraterone and enzalutamide

\begin{tabular}{lllll}
\hline & Ra-223 [33] & Cabazitaxel [34] & Abiraterone [35] & Enzalutamide [36] \\
\hline PS (ECOG) = 2 (\%) & $12^{\dagger}$ & 7 & 10 & 8.5 \\
Visceral disease (\%) & - & 25 & 18 & 23.2 \\
Bone lesions > 20 (\%) & 41 & - & - & 37.8 \\
$\geq 2$ previous chemo regimens (\%) & - & 31 & 30 & 27 \\
PD $\leq 3$ months post-docetaxel (\%) & - & 72 & 30 & - \\
PSA levels (ng/ml), median (min-max) & 146 & 143.9 & 128.8 & 107.7 \\
& $(3.8-6026)$ & $(51.1-416)$ & $(0.4-9253)$ & $(0.2-11794.1)$ \\
\hline
\end{tabular}

${ }^{\dagger}$ Patients with ECOG $\$ 2$ are included in this subgroup.

PD: Progressive disease.

An important exclusion criterion of the Ra-223 Phase III study was the presence of visceral metastases, although patients with limited lymph nodal dissemination were included [33]. On the contrary, patients with visceral metastases were enrolled in the studies with cabazitaxel (TROPIC), abiraterone (COU-AA-301) and enzalutamide (AFFIRM). Therefore, these drugs should be preferred in this setting. In particular, cabazitaxel seems to be the most indicated drug, because up to $25 \%$ patients with visceral metastases were included [34] in this study and a subgroup analysis presented in 2012 suggested a benefit for both patients with visceral metastases (OS HR =0.88; 95\% CI: 0.64-1.22) [37]. Enzalutamide, in the subgroup analysis of the AFFIRM study, seems to suggest a lower activity $(\mathrm{HR}=$ 0.78; 95\% CI: 0.56-1.09) [36]. Abiraterone, in the subset with visceral disease, achieved a median OS of 12.9 months compared with 8.3 months with placebo. Although there was a similar HR for superior survival with abiraterone in the visceral disease group, this difference did not reach statistical significance due to the much smaller sample size (HR $=0.79 ; 95 \%$ CI: $0.60-1.05 ; p=0.102$ ). The magnitude of the median survival benefit with abiraterone was similar in patients with visceral disease at baseline and those without visceral disease. Treatment with abiraterone significantly reduced the risk of radiographic progression or death by 40 and $32 \%$ in patients with visceral disease or without visceral disease, respectively [38].

Use of Ra-223 is limited to all setting of patients with symptomatic bone metastases without visceral disease independently of the previous treatment with docetaxel. In fact, the ALSYMPCA study enrolled the following three groups: patients pretreated with docetaxel, patients noneligible to docetaxel or those refusing docetaxel therapy [33]. Analysis of subgroups reported that survival benefit is similar whether or not patients had received docetaxel $(\mathrm{HR}=0.71$ vs 0.74$)[33]$.

With regard to the benefit of using Ra-223 after other therapies, currently, it is not possible to draft any conclusions about docetaxel-refractory patients, candidates to cabazitaxel, or patients previously treated with Sipuleucel-T and abiraterone or treated with two or more lines of chemotherapy. There are only available data for patients with a rapid progression to chemotherapy treated with cabazitaxel and abiraterone. In fact, the two Phase III studies, TROPIC and COU-AA-301, recruited also patients progressing less than 3 months from docetaxel [34,35].

Regarding performance status, all the studies included patients with ECOG 2. Subgroup analyses reported that the four drugs seem to induce a lower efficacy in this setting [33,34,36,38]. However, these data should be considered with caution, because the number of patients included in every single subgroup is low. With regard to pain at baseline, it is difficult to draw any conclusion, because of the different scales used and the nonavailability of full data for every single Phase III study. All analyzed Phase III studies enrolled patients with similar characteristics in terms of initial PSA level (TABLE 2). Subgroup analyses of both studies for abiraterone and enzalutamide suggest that the drugs induce benefit independently whether PSA levels are above or under the median value at baseline [36,38]. On the contrary, cabazitaxel seems to induce benefit only in the subset of patients where PSA is rising at baseline [37]. 
The analysis regarding patients treated with bisphosphonates is reported only for the ALSYMPCA study. The benefit in OS induced by Ra-223 is also similar, independent of bisphosphonates treatment $(\mathrm{HR}=0.7$ vs 0.74$)[27]$.

Patient preference is a key issue that should be evaluated prior to any drug selection; the treatment of choice should be selected after sharing the main information with the patient, and depending not only on survival and disease control, but rather on improvement in symptomatology and quality of life.

\section{Toxicity profile}

TABLE 3 summarizes the main toxicities observed in the Phase III trial conducted with Ra-223, cabazitaxel, abiraterone and enzalutamide.

Table 3. Incidence (\%) of main toxicities (grade 3-4) observed with radium-223, cabazitaxel, abiraterone and enzalutamide.

\begin{tabular}{|c|c|c|c|c|}
\hline & Radium-223 [27] & Cabazitaxel [34] & Abiraterone [35] & Enzalutamide [36] \\
\hline Anemia & 13 & 11 & 7 & - \\
\hline Neutropenic fever & - & 8 & - & - \\
\hline Thrombocytopenia & 6 & 4 & $<2$ & - \\
\hline Diarrhea & 2 & 6 & 1 & 1 \\
\hline Fatigue & - & 5 & 9 & 6 \\
\hline Hot flashes & - & - & - & 0 \\
\hline Hypertension & - & - & 1 & - \\
\hline
\end{tabular}

Data extracted from the main Phase III studies [27,34-36].

Ra-223 seems to induce slightly bone-marrow and gastrointestinal toxicities [27]. Diarrhea must be taken into consideration, since fecal excretion is the major route of elimination of the $\alpha$-emitter from the body and, consequently, fecal incontinence may compromise the metabolism of the radiopharmaceutical [27].

Cabazitaxel displays a significantly higher hematological toxicity (neutropenia, neutropenic fever, anemia and thrombocytopenia). Furthermore, cabazitaxel induced toxic deaths in 5\% of patients [34]. These data suggest that in patients with preexisting bone marrow toxicities, cabazitaxel should be used with caution, with a strict hematologic toxicity follow-up, and prophylactic G-CSF could be recommended.

Abiraterone presents an excellent tolerance profile, although it needs administration of prednisone in order to prevent the toxicity derived from the excess of mineralocorticoids due to the CYP17 blockade [35]. Less than $2 \%$ of patients presented neutropenia or severe thrombocytopenia, and $7 \%$ of patients presented grade 4 anemia. Due to the effect of mineralocorticoid, 31\% of patients presented edema/fluid retention (less than 3\% severe), $17 \%$ hypokalemia (less $4 \%$ severe) and $10 \%$ hypertension (1\% severe). Overall, abnormalities in liver function tests occurred at a similar frequency in the abiraterone acetate and placebo groups, including changes of any grade in liver function tests (10 and 8\%, respectively) and grade 3 or 4 changes in liver function tests (3.5 and 3.0\%) [35]. 
Finally, enzalutamide showed a toxicity profile similar to placebo; the main side effects of enzalutamide were fatigue $(6 \%$, grade 3$)$, diarrhea (1\%, grade 3$)$, hot flushes $(20 \%$, all grade $1-2)$, bone pain $(1 \%$, grade 3$)$ and headache $(<1 \%$, severe); cardiac events were observed in $6 \%$ of patients with enzalutamide versus $8 \%$ in the placebo arm, with a slightly higher hypertension incidence in the experimental arm (6.6 vs 3.3\%). No evidence of metabolic syndrome was observed [38]. It is worth mentioning that the AFFIRM study was amended, excluding patients with seizures due to a supposed induction of sudden attack by enzalutamide [36].

\section{New methods of evaluation}

In addition to providing clinical information, studies conducted with Ra-223 also provided information regarding new possible methods of biochemical (BAP) and radiological follow-up Positron Emission Tomography (PET) for patients on therapy.

\section{Bone alkaline phosphatase}

A frequent diagnostic open issue in the field of prostate cancer is related to the directional changes of PSA levels, the most frequently altered biomarker in the disease.

It is known that PSA can reflect the burden of CRPC [39], and consequently, monitoring PSA changes could support in detecting reduction in disease burden after treatment. However, the interpretation of PSA changes seems to be affected by several important caveats, as those correlated to the evaluation of response after immunological or cytostatic treatment [40]. In this regard, the Prostate Cancer Working Group guidelines do not recognize PSA changes as the sole endpoint to decide change of therapy or to declare treatment failure, so that further biomarkers are needed [40].

Investigations on Ra-223 conducted since Phase I study revealed that its activity was related to the reduction of BAP [41,42]. According to investigators of ALSYMPCA study, BAP appeared as a more sensible biomarker than the classic PSA; therefore, BAP was evaluated as secondary endpoint through the following three parameters: time to total BAP progression, total BAP response and total BAP normalization [27]. Future studies could confirm the important role of BAP in the follow-up of patients with bone metastases treated with the other drugs for prostate cancer. More evidence is needed but, with the results obtained from the ALSYMPCA trial, it could be recommended to perform BAP assessments and take into consideration with both imaging procedures and PSA. A predictive model including BAP, PSA and eventually circulating tumoral cells may be of interest in this set of patients; further prospective studies are a medical need.

\section{${ }^{18}$ F-fluoride PET}

A second diagnostic point emerging from Ra-223 studies is related to radiological imaging monitoring of bone metastases. Bone metastases are difficult to monitor during treatment, and the imaging modalities used to assess disease in bone have not been standardized. The $99 \mathrm{mTc}$-methylene diphosphonate scintigraphy is a traditional diagnostic therapy with well-known limits of sensitivity. A qualitative assessment of conventional bone scintigraphy with $99 \mathrm{mTc}$ methylene diphosphonate is perceived as an insensitive method for monitoring the treatment response of bone metastases.

\footnotetext{
${ }^{18}$ F-fluoride PET/CT is a highly sensitive and specific modality for detection of bone metastases in patients with high-risk prostate cancer. It is more specific than ${ }^{18} \mathrm{~F}$-fluoride PET alone and more sensitive and specific than single-photon emission computed tomography (SPECT) and bone scintigraphy. Detection of bone metastases is improved by SPECT compared with planar bone scintigraphy, and by ${ }^{18}$ F-fluoride PET compared with SPECT [43]. Recently, a pilot study was conducted to evaluate the
} 
efficacy of early treatment response to Ra-223 with ${ }^{18} \mathrm{~F}$-fluoride PET [44]. This study showed the feasibility of measuring variation in the uptake of ${ }^{18} \mathrm{~F}$-fluoride with PET during the treatment with Ra-223 and suggested that the semi-quantitative ${ }^{18} \mathrm{~F}$-fluoride PET is more accurate than the qualitative comparison of scans in assessing response in bone metastases. In fact, a correlation with the PSA response and BAP activity was observed, offering a potential imaging biomarker for monitoring treatment response in bone metastases following treatment with Ra-223. Studies with more patients are needed to establish a formal method of analysis and provide better diagnostic and prognostic information.

Nevertheless, the available data with regard to the use of ${ }^{18} \mathrm{~F}$-fluoride PET in this setting is very limited so far. The study performed in patients with high-risk prostate cancer included a very small number of patients, and therefore limitations of the data are clearly present. Thus, PET would be recommended to be added to the suggested algorithm, including PSA, BAP and imaging tests.

\section{Ongoing \& future studies}

The achieved outcomes so far with Ra-223 encourage fulfilling further steps in the studies to better understand the activity of this radiopharmaceutical and to try to extend its benefit. Currently, clinical development is addressed in the following directions:

- Continue testing its efficacy and adverse effects on expanded access studies.

- Explore different administration schedules and their use in different patient populations.

- Combination with other agents.

Two prospective, interventional, open-label, multicenter early access program studies are ongoing $[45,46]$. Both are being conducted in CRPC patients diagnosed with bone metastases, with the collection of additional short- and long-term safety data of Ra-223. Inclusion and exclusion criteria for patients are similar to those of the Phase III trial ALSYMPCA.

As far as different schedule of treatment is concerned, data from reported Phase I and II studies are promising with regard to safety and tolerability, with studying Ra-223 at the dose of $50 \mathrm{kBq} / \mathrm{kg}$ administered at 4 weekly intervals [31,32]. Therefore, both dose and schedule were chosen for the experimental arm in the ALSYMPCA trial. However, a dose response in the BAP decrease according to different doses of Ra-223 has been observed, and different intervals between administrations have been also studied (3-4 weeks) [27,33,34]. This implicates the need to explore new schedules based on different dose escalations and treatment duration. In addition, we need to determine the optimal interval between doses $(3,4$ or 6 weeks) and the total number of administrations that are associated with an optimum benefit.

Similarly, as with other drugs (e.g., docetaxel), another interesting question is, whether there is a role for re-treatment with Ra-223 in patients who benefit from the first treatment.

Due to the singular mechanism of action, it was reported that there exists a strong rationale for the combination of Ra-223 with the main antitumor drugs already approved for the treatment of CRPC patients, such as docetaxel, cabazitaxel, abiraterone acetate and enzalutamide. These agents, in fact, could complement the action of Ra-223, allowing its use in patients with bone metastases.

Concerning the association with chemotherapy, a Phase I-II study is trying to establish the recommended dose of Ra-223 to be used in combination with docetaxel in patients with metastatic CRPC, and to investigate the safety and efficacy of this combination [47]. The first step of the study concluded that dose regimen of Ra-223 $(50 \mathrm{kBq} / \mathrm{kg} \mathrm{q} 6$ weeks $\times 5)$ combined with docetaxel $\left(60 \mathrm{~g} / \mathrm{m}^{2} \mathrm{q} 3\right.$ weeks $\times$ 10 ) is safe, allowing to proceed into the following Phase IIa, in which this combination will be compared with docetaxel $\left(75 \mathrm{mg} / \mathrm{m}^{2}\right)$ every 3 weeks. 
Combination studies with either abiraterone or enzalutamide are encouraging on account of their very low toxicity profile, but there data are not yet available to support either of these combinations. These combinations are being tested currently in several trials; two of them are particularly interesting:

A Phase III, double-blinded, placebo-controlled, multinational trial will randomize 800 patients with asymptomatic or mildly symptomatic, chemotherapy-naïve, bone-predominant metastatic CRPC to receive $\mathrm{Ra}-223$ (50 kBq/kg iv.) every 4 weeks for six cycles or Ra-223 plus abiraterone, followed by abiraterone until a symptomatic skeletal event or death occurs [48]. The primary objective is progressionfree survival (PFS). Secondary endpoints include OS, time to pain progression, time to opiate use for cancer pain, time to cytotoxic chemotherapy, radiographic progression-free survival and acute and longterm safety. This trial is currently recruiting participants.

On the other hand, a randomized Phase III trial comparing enzalutamide versus Ra-223 and enzalutamide in asymptomatic or mildly symptomatic patients metastatic to the bone will recruit 560 patients. The objective of this trial is to assess if upfront combination of enzalutamide and Ra-223 improves radiological progression-free survival compared to single agent enzalutamide [49].

\section{Expert commentary}

The knowledge about prostate cancer therapeutics and prognostic- predictive factors has been increasing in the recent years, and several new drugs have been added to the previously available treatments. Nevertheless, there is not a clear algorithm about treatment line choices, and there is no solid data yet to strongly recommend the procedures to be performed in order to evaluate disease progression.

ALSYMPCA trial aimed to include a very wide population to get applicable results to routine clinical practice, and all subgroups achieved clinical benefit with Ra-223, including the group of patients that had received prior docetaxel. Docetaxel is still a treatment option in this kind of patients, despite many physicians trying to avoid chemotherapy in patients who are only presenting bone metastases.

\section{Five-year view}

Probably the key results will be given as soon as there is more data from the trials combining Ra-223 and abiraterone or enzalutamide. Also, a trial testing the combination of Ra-223 plus docetaxel in patients with both bone and visceral metastases could clarify some aspects that are still unanswered. How to deal with those patients with bone and visceral disease? Which would be the best treatment sequence? The more information is got, the more questions appear.

Future research will be necessary to clarify further benefits associated with different treatment schedules (e.g., different number of doses, frequency of administration) or the possibility of re-treating patients who previously and successfully responded to this therapy, but probably Ra-223 will be a standard combined with other treatments, as other chemotherapeutic or hormonal agents in order to expand its benefit in CRPC patients with bone metastases.

\section{Conclusions}

The hypothesis that $\alpha$-emitters could represent a new paradigm in the treatment of prostate cancer has been confirmed by the use of Ra-223. Ra-223 induced a significant survival benefit, delaying the onset of SRE and showing an acceptable bone marrow safety profile, and all these achievements had never been seen described using the previously available radiopharmaceuticals. In particular, its survival benefit appears comparable to that described with chemotherapeutic and hormonal agents in a similar subset of 
patients. New opportunities have emerged after the study with Ra-223, such as new biochemical markers and new imaging methods.

\section{Financial \& competing interests disclosure}

Funding for this study was provided by Bayer HealthCare. Sonia Maciá (Pivotal S.L) provided advice to properly handle and submit the paper. The authors have no relevant affiliations or financial involvement with any organization or entity with a financial interest in or financial conflict with the subject matter or materials discussed in the manuscript. This includes employment, consultancies, honoraria, stock ownership or options, expert testimony, grants or patents received or pending, or royalties.

No writing assistance was utilized in the production of this manuscript.

\section{References}

Papers of special note have been highlighted as: $\bullet$ of considerable interest

1. Coleman RE. Skeletal complications of malignancy. Cancer 1997;80:1588-94

2. Jimenez-Andrade JM, Mantyh WG, Bloom AP, et al. Bone cancer pain. Ann N Y Acad Sci 2010;1198:173-81

3. Saylor PJ, Smith MR. Bone health and prostate cancer. Prostate Cancer Prostatic Dis 2010;13:20-7

4. Coleman RE. Clinical features of metastatic bone disease and risk of skeletal morbidity. Clin Cancer Res 2006;12:6243s-9s

5. $\quad$ Adami S. Bisphosphonates in prostate carcinoma. Cancer 1997;80(8 Suppl):1674-9

6. Silberstein EB. Systemic radiopharmaceutical therapy of painful osteoblastic metastases. Semin Radiat Oncol 2000;10:240-9

7. Fizazi K, Lipton A, Mariette X, et al. Randomized phase II trial of denosumab in patients with bone metastases from prostate cancer, breast cancer, or other neoplasms after intravenous bisphosphonates. J Clin Oncol 2009;27:1564-71

8. Lutz S, Berk L, Chang E, et al. Palliative radiotherapy for bone metastases: an ASTRO evidence-based guideline. Int J Radiat Oncol Biol Phys 2011;79:965-76

9. Robinson RG, Preston DF, Schiefelbein M, Baxter KG. Strontium 89 therapy for the palliation of pain due to osseous metastases. JAMA 1995;274:420-4

10. Serafini AN, Houston SJ, Resche I, et al. Palliation of pain associated with metastatic bone cancer using samarium-153 lexidronam: a double-blind placebo-controlled clinical trial. J Clin Oncol 1998;16:157481

11. Maxon HRIII, Schroder LE, Hertzberg VS, et al. Rhenium-186(Sn)HEDP for treatment of painful osseous metastases: results of a double-blind crossover comparison with placebo. J Nucl Med 1991;32:1877-81

12. Atkins HL. Overview of nuclides for bone pain palliation. Appl Radiat Isot 1998;49:277-83

\section{•- A review about main radiopharmaceuticals used for systemic therapy for pain relief.}

13. Srivastava SC, Atkins HL, Krishnamurthy GT, et al. Treatment of metastatic bone pain with tin- $117 \mathrm{~m}$ Stannic diethylenetriaminepentaacetic acid: a phase I/II clinical study. Clin Cancer Res 1998;4:61-8

14. Bouchet LG, Bolch WE, Goddu SM, et al. Considerations in the selection of radiopharmaceuticals for palliation of bone pain from metastatic osseous lesions. J Nucl Med 2000;41:682-7

15. Bodei L, Lam M, Chiesa C, et al. EANM procedure guideline for treatment of refractory metastatic bone pain. Eur J Nucl Med Mol Imaging 2008;35:1934-40

16. Mulford D, Scheinberg D, Jurcic J. The promise of targeted $\alpha$-particle therapy. J Nucl Med 2005;46:199S-204S

17. Tiepolt C, Gruning T, Franke WG. Renaissance of 224 Ra for the treatment of ankylosing spondylitis: clinical experiences. Nucl Med Commun 2002;23:61-6

18. Nilsson S, Strang P, Aksnes AK, et al. A randomized, dose-response, multicenter phase II study of radium-223 chloride for the palliation of painful bone metastases in patients with castration-resistant prostate cancer. Eur J Cancer 2012;48:678-86

19. Parker C, Nilsson S, Heinrich D, et al. Alpha emitter radium-223 and survival in metastatic prostate cancer. N Engl J Med 2013;369:213-23 
20. Henriksen G, Breistol K, Bruland OS, et al. Significant antitumor effect from bone-seeking, alphaparticle-emitting (223)Ra demonstrated in an experimental skeletal metastases model. Cancer Res 2002;62:3120-5

21. McDevitt MR, Sgouros G, Finn RD. Radioimmunotherapy with alpha-emitting nuclides. Eur J Nucl Med 1998;25:1341-51

22. Bruland OS, Nilsson S, Fisher DR, Larsen RH. High-linear energy transfer irradiation targeted to skeletal metastases by the alpha-emitter 223Ra: adjuvant or alternative to conventional modalities? Clin Cancer Res 2006;12:6250s-7s

23. .• A helpful article to understand radium-223 (Ra-223) mechanism of action.

24. Henriksen G, Fisher DR, Roeske JC, et al. Targeting of osseous sites with alpha-emitting 223 Ra: comparison with the beta-emitter 89Sr in mice. J Nucl Med 2003;44:252-9

25. Lewington VJ. Bone-seeking radionuclides for therapy. J Nucl Med 2005;46(Suppl 1):38S-47S

26. Dornish M, Heier-Baardson H, Petterson EO. Cellular effects of alpha particle radiation from radium223: ALPHARADIN, a new radiopharmaceutical for the treatment of skeletal metastases. Proc Am Assoc Canc Res (AACR) 2008;49

27. Nilsson S, Franzen L, Parker C, et al. Two-year survival follow-up of the randomized, double-blind, placebo-controlled phase II study of radium-223 chloride in patients with castration-resistant prostate cancer and bone metastases. Clin Genitourin Cancer 2013;11:20-6

28. - A paper to understand the safety profile within 2 years in the patients included at the Phase II trial.

29. Nilsson S, et al. 1.5-year post-treatment follow-up of radium-223 dichloride (Ra-223) in patients with castration-resistant prostate cancer (CRPC) and bone metastases from the phase 3 ALSYMPCA study. J Clin Oncol 2014;32(Suppl 4):Abstract 9

30. Nilsson S, Larsen RH, Fossa SD, et al. First clinical experience with alpha-emitting radium-223 in the treatment of skeletal metastases. Clin Cancer Res 2005;11:4451-9

•- The first experience with Ra-223 in patients, which showed a very interesting safety profile.

31. Nilsson S BL, Fosså SD, Westling JE, et al. Phase I study of alpharaldin ${ }^{\mathrm{TM}}$ (223Ra), an $\alpha$-emitting bone-seeking agent in cancer patients with skeletal metastases. Eur J Nucl Med Mol Imaging 2004;31

32. .• Other Phase I trial to add more data regarding safety about Ra-223.

33. Nilsson S, Franzen L, Parker C, et al. Bone-targeted radium-223 in symptomatic, hormone-refractory prostate cancer: a randomised, multicentre, placebo-controlled phase II study. Lancet Oncol 2007;8:587-94

\section{4. •• Positive efficacy results in a Phase II study, matching primary and secondary end points}

35. Parker CC, Pascoe S, Chodacki A, et al. A randomized, double-blind, dose-finding, multicenter, phase 2 study of radium chloride ( $\mathrm{Ra} 223$ ) in patients with bone metastases and castration-resistant prostate cancer. Eur Urol 2013;63:189-97

36. Parker C, Coleman R, Nilsson S, et al. Update survival, quality of life (QOL), and safety data of Radium-223 chloride (223Ra) in patients with castration-resistant prostate cancer (CRPC) with bone metastases from the phase 3 double-blind, randomized, multinational study (ALSYMPCA). ESMO 2012; Oct 2:poster 898 Annals of Oncology supp 2012

37. • Very solid results comparing Ra-223 plus best standard of care versus placebo plus best standard of care.

38. Sartor O, Coleman R, Nilsson S, et al. Effect of radium-223 dichloride on symptomatic skeletal events in patients with castration-resistant prostate cancer and bone metastases: results from a phase 3, doubleblind, randomised trial. Lancet Oncol 2014;15(7):738-46

39. de Bono JS, Oudard S, Ozguroglu M, et al. Prednisone plus cabazitaxel or mitoxantrone for metastatic castration-resistant prostate cancer progressing after docetaxel treatment: a randomised open-label trial. Lancet 2010;376:1147-54

40. de Bono JS, Logothetis CJ, Molina A, et al. Abiraterone and increased survival in metastatic prostate cancer. N Engl J Med 2011;364:1995-2005 
41. Scher HI, Fizazi K, Saad F, et al. Increased survival with enzalutamide in prostate cancer after chemotherapy. N Engl J Med 2012;367:1187-97

42. Oudard S, de Bono J, Ozguroglu M, et al. Impact of cabazitaxel (Cbz) + prednisone (P; CbzP) on overall survival (OS) at 2 yrs and in patients (pts) with aggressive disease: post-hoc analyses of TROPIC trial. Presented at European Society for Medical Oncology; 28 September - 2 October 2012; Vienna, Austria

43. Goodman OBJr, Flaig TW, Molina A, et al. Exploratory analysis of the visceral disease subgroup in a phase III study of abiraterone acetate in metastatic castration-resistant prostate cancer. Prostate Cancer Prostatic Dis 2014;17:34-9

44. Vollmer RT, Dawson NA, Vogelzang NJ. The dynamics of prostate specific antigen in hormone refractory prostate carcinoma: an analysis of cancer and leukemia group B study 9181 of megestrol acetate. Cancer 1998;83:1989-94

45. Armstrong AJ, Eisenberger MA, Halabi S, et al. Biomarkers in the management and treatment of men with metastatic castration-resistant prostate cancer. Eur Urol 2012;61:549-59

46. Coleman RE, Major P, Lipton A, et al. Predictive value of bone resorption and formation markers in cancer patients with bone metastases receiving the bisphosphonate zoledronic acid. J Clin Oncol 2005;23:4925-35

47. Jung K, Miller K, Wirth M, et al. Bone turnover markers as predictors of mortality risk in prostate cancer patients with bone metastases following treatment with zoledronic acid. Eur Urol 2011;59:60412

48. Even-Sapir E, Metser U, Mishani E, et al. The detection of bone metastases in patients with high-risk prostate cancer: 99mTc-MDP Planar bone scintigraphy, single- and multi-field-of-view SPECT, 18Ffluoride PET, and 18F-fluoride PET/CT. J Nucl Med 2006;47(2):287-97

49. Cook GJr, Parker C, Chua S, et al. 18F-fluoride PET: changes in uptake as a method to assess response in bone metastases from castrate-resistant prostate cancer patients treated with 223Ra-chloride (Alpharadin). EJNMMI research 2011;1:4

50. •• It explains how 18F-fluoride PET can provide better information about disease response compared with other imaging tests.

51. Radium-223 Dichloride (BAY88-8223) in Castration-Resistant (Hormone-Refractory) Prostate Cancer Patients With Bone Metastases [Internet]. 2012. Available from: http://clinicaltrials.gov/show/NCT01516762

52. Radium(223) Dichloride (Alpharadin) in Castration-Resistant (Hormone-Refractory) Prostate Cancer Patients With Bone Metastases. [Internet]. 2012. Available from: http://clinicaltrials.gov/show/NCT01618370

53. A Study of Alpharadin ${ }^{\circledR}$ With Docetaxel in Patients With Bone Metastasis From Castration-Resistant Prostate Cancer (CRPC). [Internet]. 2010. Available from: http://clinicaltrials.gov/show/NCT01106352

54. Smith M, Parker C, Tombal B, et al. ERA 223-A phase 3 trial of radium-223 dichloride (Ra-223) in combination with abiraterone acetate (AA) and prednisone in the treatment of asymptomatic or mildly symptomatic chemotherapy-naïve patients with bone-predominant metastatic castration-resistant. Ann Oncol 2014;25(Suppl 4):iv278

55. Phase III Radium 223 mCRPC-PEACE III. Available from: http://clinicaltrials.gov/show/NCT02194842 